\title{
Survival Rate of Patients with Gastric Cancer and effective factors in two referral hospitals of Mashhad, Iran: A Multiple Regression Analysis
}

\author{
Zahra Yousefli ${ }^{1}$, Ladan Goshayeshi ${ }^{2}$, Hamid Heidarian Miri ${ }^{3}$, Naiemeh Farhangnezhad ${ }^{1}$, Aida Ghofrani ${ }^{1}$, Zahra \\ Dashti $^{1}$, Ramin Hoseinzadeh ${ }^{1}$
}

${ }^{1}$ Gastrology and Hepatology Research Center, Faculty of Medicine, Mashhad University of Medical Sciences, Mashhad, Iran

${ }^{2}$ Assistant Professor, Department of Gastrology and Hepatology, Faculty of Medicine, Mashhad University of Medical Sciences, Mashhad, Iran

${ }^{3}$ Assistant Professor, Department of Epidemiology and Biostatistics, School of Public Health, Mashhad University of Medical Sciences, Mashhad, Iran

\section{Type of article: Original}

\begin{abstract}
Background: Gastric cancer (GC) is among the most prevalent cancers in both genders, and the survival rate constitutes a measure to evaluate the diagnosis and treatment of GC. Mashhad is a city located in the northern part of Iran where has been identified as a high incidence region for GC.

Objective: To determine survival rate of GC patients diagnosed in Mashhad and identify whether underling factors including demographic factors, family history, education, addiction and co-morbidities can affect survival rate.

Methods: This cross-sectional study was conducted in two tertiary referral hospitals (Ghaem Hospital and Imamreza Hospital) in Mashhad. Patients were all diagnosed with GC from 2012 to 2016, and their status in 2019 was determined by data collected from the patients' records in the hospitals and also by phone calls. KaplanMeier, Log-Rank test, and Cox Proportional Hazards Model, were used for data analysis using STATA 14.1 software.

Results: Among 308 patients, 230 (74.7\%) were male, and 78 (25.3\%) were female. The mean of patients' age and age at diagnosis time were 65.49 and 64.72 years, respectively. Survival proportion at first, third, and fifth year after diagnosis in both sexes using Kaplan-Meier were 0.63, 0.20, and 0.16. Univariate analysis showed that ethnicity $(\mathrm{HR}=1.58, \mathrm{p}=0.034)$, addiction $(\mathrm{HR}=0.53, \mathrm{p}=0.016)$, positive family history $(\mathrm{HR}=1.56, \mathrm{p}=0.023)$, past medical history $(\mathrm{HR}=7.93, \mathrm{p}=0.002)$ and education level $(\mathrm{HR}=0.44, \mathrm{p}=0.045)$ had significant effect on survival rate. Multivariate analysis was somehow consistent with those results except for education level. $(\mathrm{HR}=9.33$, $\mathrm{p}=0.013$ ).

Conclusion: Older age at diagnosis time, male sex, positive family history, coronary artery disease, diabetes mellitus, and hypertension can negatively influence the survival rate of gastric cancer patients, and these could be applied for improving the GC screening and treatment guidelines.
\end{abstract}

Keywords: Mashhad, Proportional Hazards Models, Stomach Neoplasms, Survival Rate

\section{Abbreviations / Acronyms:}

ASR: Age-standardized Rate, CAD: Coronary Artery Disease, DM: Diabetes Mellitus, GC: Gastric Cancer; HR: Hazard Ratio, HTN: Hypertension

\section{Corresponding author:}

Assistant Professor Dr. Ladan Goshayeshi. Department of Gastrology and Hepatology, Faculty of Medicine, Mashhad University of Medical Sciences, Mashhad, Iran.

Tel: +98.9155109704, Fax: +98.5138932481, E-mail: Goshayeshil@mums.ac.ir

Received: December 30, 2020, Accepted: April 29, 2021, Published: June 2021

Funding / research project approval: Mashhad University of Medical Sciences (Grant no: 941320)

Ethics approval: Ethics committee of Mashhad University of Medical Sciences (Ref.: IR.MUMS.REC.1395.143)

(C) 2021 The Authors. This is an open access article under the terms of the Creative Commons Attribution-NonCommercialNoDerivs License, which permits use and distribution in any medium, provided the original work is properly cited, the use is non-commercial and no modifications or adaptations are made. 


\section{Introduction}

Gastric cancer is reported as the fifth most frequently diagnosed malignancy in the world, with approximately 783,000 deaths annually, which accounts for $8.2 \%$ of total cancer deaths. Gastric cancer is the third leading cause of cancer death in both sexes, and incidence rates are two-fold greater in men than women (1). Unlike Japan, Western Europe, and North America, the Gastric cancer incidence rate has been rising over the past 30 years in Iran (2-4). According to the latest statistics of the Iran Cancer Research Center, Gastric cancer is the most common cancer among Iranian men and the third most common cancer among Iranian women after breast cancer (5).

Regarding gastric cancer incidence rate, various geographical regions in the world can be divided into three regions: high-incidence regions (Age-standardized Rate: ASR $>20$ ), middle-incidence regions (ASR $>10$ ), and low-incidence regions $(\mathrm{ASR}<10)(6)$. In Iran, northern, central, and western regions were identified as high, middle, and low incidence regions, respectively $(2,7,8)$ and since Mashhad is located in the northeast part of Iran, the incidence rate is high. The 5-year survival rate is one of the most important objectives measured in order to evaluate the diagnosis and treatment for patients diagnosed with Gastric cancer (9). The 5-year survival rate is relatively low in patients with Gastric cancer even after surgery, due to the fact that most patients with Gastric cancer are diagnosed in a stage in which conventional therapies such as gastrostomy, chemotherapy, or radiation therapy are not effective to increase the patients' survival (10).

In recent years, many studies were conducted in different provinces of Iran on gastric cancer to estimate the survival rate of these patients. Veisani et al. has reviewed all these studies and published them as a systematic review (11). However, that review revealed no data from Khorasan-Razavi province. In our previous study, we designed a predictive model of survival time for patients suffering from gastric cancer by data mining techniques using genetic algorithms and logistic regression (12). Nevertheless, the current study was carried out to determine the 1, 3, and 5year survival rates in patients with Gastric cancer using available data. These patients were diagnosed and undergone treatment at two main tertiary referral hospitals in Mashhad in Khorasan-Razavi province to determine whether demographic factors, including age, gender, living place, education and past medical history, family history affect patients' survival rate.

\section{Material and Methods}

\subsection{Research design}

This retrospective cross-sectional study was conducted to determine whether demographic factors and past medical history might affect the survival rate of patients diagnosed with gastric cancer in Mashhad.

\subsection{Study population}

The study population includes all available patients diagnosed with gastric cancer in two main tertiary referral hospitals, Ghaem and Imamreza, from 2012 to 2016. These patients had records in archives of the hospitals about their diagnosis, age at diagnosis time, pathology, and also their addresses and phone numbers were available for subsequent follow-ups. Inclusion criteria was gastric cancer, which was confirmed by biopsy in patients of any age who had agreed to participate in the study by filling informed consent. Any patients with gastric cancer who were not residents of Khorasan Razavi province, the patients who had died for any reason other than gastric cancer, and those who were suffering from two or more primary cancers were excluded from the study.

\subsection{Data collection}

The survival status of these patients in 2019 was determined by the data collected from available archived files in the hospitals and also by phone calls to the patients or, in case of the patient's death, their relatives for subsequent follow-ups and to determine the exact time of death. The survival time, including first, third, and fifth year, was measured for all patients who were not alive at the end of the study period.

\subsection{Statistical analysis}

The effect of demographic and clinical variables such as gender, age at the time of diagnosis, addiction, ethnicity, level of education, family history, and past medical history was studied. Data were put in STATA 14.1 software for statistical indexes, and analysis was performed using Kaplan - Meier, Log- Rank Test, and Cox Proportional Hazards Model. The significance level was considered less than 0.05 . 


\subsection{Research ethics}

Ethics approval was obtained from the ethical committee of Mashhad University of Medical Sciences (IR.MUMS.REC.1395.143), and patients' information was kept confidential during the study.

\section{Results}

\subsection{Demographic profile}

Among 308 patients 230 (74.7\%) were male, 78 (25.3\%) were female, 35 (23.18\%) were addicted and 117 (61.58\%) were illiterate. The mean patients' age was 65.49 years. The mean and median age at diagnosis time were 64.72 and 66 years, respectively. Table 1 shows the demographic characteristics of participants in detail.

Table 1. Patients' profiles

\begin{tabular}{|l|l|l|l|}
\hline Variable & $\mathrm{n}$ & $\%$ \\
\hline \multirow{3}{*}{ Gender } & Male & 230 & 74.7 \\
\cline { 2 - 4 } & Female & 78 & 25.3 \\
\hline Pathology & Non-adenocarcinoma & 33 & 12.18 \\
\cline { 2 - 4 } & Adenocarcinoma & 238 & 87.82 \\
\hline Ethnicity & Fars & 142 & 78.45 \\
\cline { 2 - 4 } & Others & 39 & 21.55 \\
\hline Addiction & No & 116 & 76.82 \\
\cline { 2 - 4 } & Yes & 35 & 23.18 \\
\hline Past medical history & HTN & 34 & 44.16 \\
\cline { 2 - 4 } & CAD & 16 & 20.78 \\
\cline { 2 - 4 } & DM & 10 & 12.99 \\
\cline { 2 - 4 } & DM +HTN & 6 & 7.79 \\
\cline { 2 - 4 } & DM+HTN+CAD & 4 & 5.19 \\
\cline { 2 - 4 } & HTN+CAD & 7 & 9.09 \\
\hline Family history of gastric cancer & No & 200 & 64.94 \\
\cline { 2 - 4 } & Yes & 108 & 35.06 \\
\hline Education level & Illiterate & 117 & 61.58 \\
\cline { 2 - 4 } & Less than high school diploma & 59 & 31.05 \\
\cline { 2 - 4 } & High school diploma or higher & 14 & 7.37 \\
\hline
\end{tabular}

\subsection{Univariate and Multiple regression analyses}

The survival proportion at first, third, and fifth years after diagnosis of gastric cancer in both sexes using KaplanMeier was $0.63,0.20$, and 0.16 , respectively (Table 2, Figure 1). The mean and median of follow-up time were 15.91 and 13.53 months, in turn. Each potentially related variable was set as an independent variable in the Cox proportional hazard regression model in the univariate analysis. The univariate analysis showed that the Hazard Ratios (HR) of death in patients in an ethnic population other than Fars is 1.58 times greater than Fars ( $\mathrm{p}=0.034)$. The results also showed that addiction might decrease death hazard by 0.53 times $(p=0.016)$. Moreover, in the univariate analysis hazard ratio of death for patients with a high school diploma or higher level of education was 0.44 times lesser than those who were illiterate $(\mathrm{p}=0.045)$. While, older age at diagnosis time, positive family history of GC and past medical history including hypertension, coronary artery disease, and diabetes mellitus can increase the hazard ratio of death by $1.05(\mathrm{p}<0.001), 1.56(\mathrm{p}=0.023)$, and $7.93(\mathrm{p}=0.002)$, respectively.

Table 2. Survival proportion of patients with gastric cancer at Imam Reza Hospital

\begin{tabular}{|l|l|l|}
\hline Duration & Survival proportion & $95 \%$ Confidence Interval \\
\hline One year & 0.63 & $0.54-0.70$ \\
\hline Three year & 0.20 & $0.13-0.27$ \\
\hline Five year & 0.16 & $0.09-0.23$ \\
\hline
\end{tabular}

In multiple regression analysis, sex was shown to be a strong predictor of survival as well as other variables with a p-value considered less than 0.25 to enter in the regression model. This means women had a higher survival rate than men by 2.08 times $(\mathrm{p}=0.094)$. Proportional-hazards assumption was shown to be met using the Schoenfeld residuals test ( $>0.9)$. Results of multiple Cox proportional hazard regression models showed that after adjusting 
other variables, variables including age at diagnosis time, comorbidities like diabetes mellitus (DM), hypertension (HTN), coronary artery disease (CAD) could be related to the survival of patients with gastric cancer. Meaning, after adjusting other variables, the hazard of death at any age was 1.07 times greater than the preceding year; thus, survival of younger patients is higher than older ones. Besides, the hazard ratio of death in patients with HTN + CAD and $\mathrm{DM}+\mathrm{HTN}+\mathrm{CAD}$ relative to patients with only blood hypertension was 3.22 and 7.35 times higher, respectively. Table 3 shows univariate and multivariate analysis in detail.

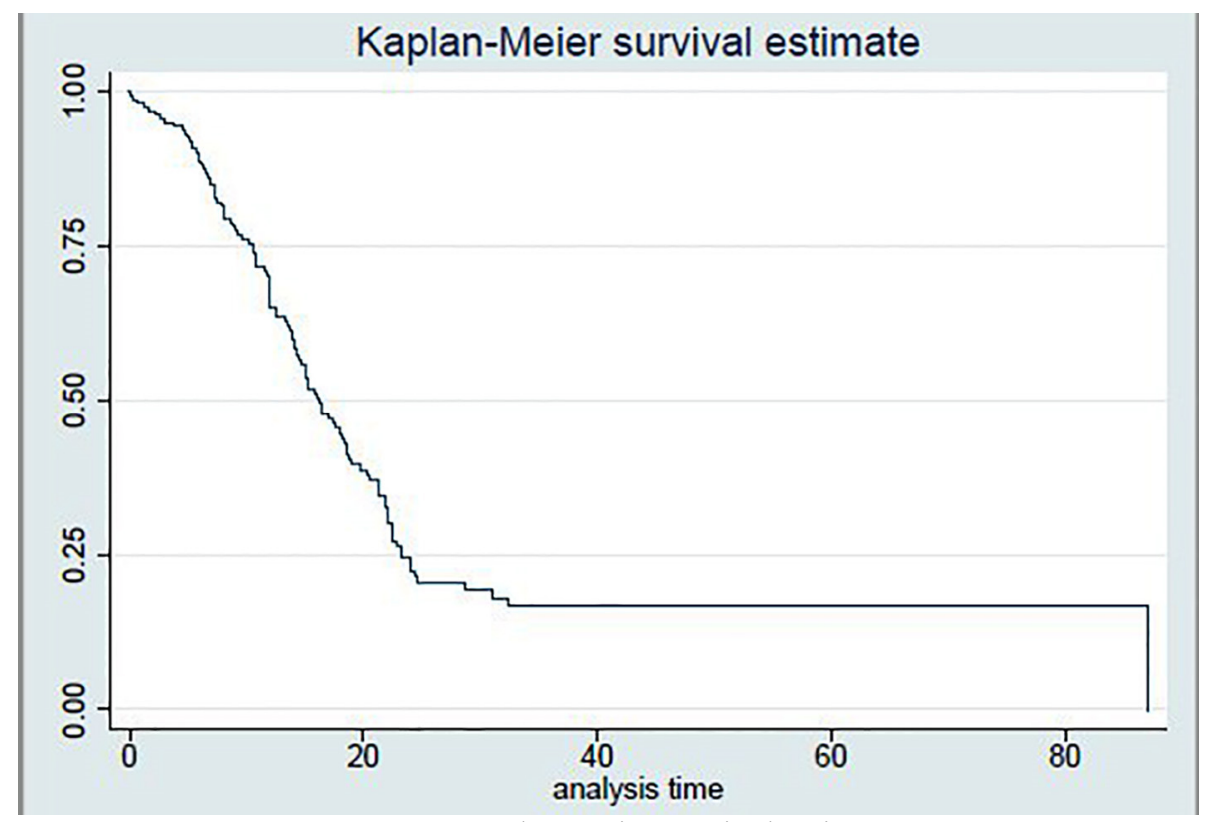

Figure 1. Kaplan-Maier survival estimate

Table 3. Univariate and Multivariate analysis of Potential Risks factors

\begin{tabular}{|c|c|c|c|c|c|c|c|}
\hline \multicolumn{2}{|l|}{ Variable } & \multicolumn{3}{|c|}{ Univariate regression analysis } & \multicolumn{3}{|c|}{ Multiple regression analysis } \\
\hline & & $\begin{array}{l}\text { Hazard } \\
\text { Ratio }\end{array}$ & $\mathrm{p}$-value & $95 \% \mathrm{CI}$ & $\begin{array}{l}\text { Hazard } \\
\text { Ratio }\end{array}$ & $\mathrm{p}$-value & $95 \% \mathrm{CI}$ \\
\hline Gender & Women/Men & 0.75 & 0.233 & $(0.48-1.19)$ & 0.48 & 0.094 & $(0.20-1.13)$ \\
\hline Ethnicity & Others/Fars & 1.58 & 0.034 & $(1.03-2.42)$ & 1.56 & 0.273 & $(0.70-3.49)$ \\
\hline Addiction & Yes/No & 0.53 & 0.016 & $(0.32-0.89)$ & 0.52 & 0.153 & $(0.21-1.27)$ \\
\hline \multicolumn{2}{|c|}{ Age at diagnosis time } & 1.05 & 0.000 & $(1.03-1.08)$ & 1.07 & 0.001 & $(1.02-1.12)$ \\
\hline \multirow{6}{*}{$\begin{array}{l}\text { Past medical } \\
\text { history }\end{array}$} & Hypertension (HTN) & \multicolumn{6}{|c|}{ Reference } \\
\hline & Coronary artery disease (CAD) & 0.95 & 0.907 & $(0.45-2.00)$ & 1.13 & 0.763 & $(0.49-2.63)$ \\
\hline & diabetes mellitus (DM) & 0.43 & 0.096 & $(0.16-1.15)$ & 0.50 & 0.286 & $(0.14-1.78)$ \\
\hline & $\mathrm{DM}+\mathrm{HTN}$ & 2.83 & 0.041 & $(1.04-7.71)$ & 2.26 & 0.168 & $(0.70-7.21)$ \\
\hline & $\mathrm{HTN}+\mathrm{CAD}$ & 5.21 & 0.002 & $(1.80-15.08)$ & 3.22 & 0.043 & $(1.03-9.98)$ \\
\hline & $\mathrm{DM}+\mathrm{HTN}+\mathrm{CAD}$ & 7.93 & 0.002 & $(2.16-29.04)$ & 7.35 & 0.005 & $(1.83-29.53)$ \\
\hline $\begin{array}{l}\text { Family } \\
\text { history }\end{array}$ & Yes/No & 1.56 & 0.023 & $(1.06-2.30)$ & 1.59 & 0.09 & $(0.72-3.49)$ \\
\hline \multirow[t]{3}{*}{ Education level } & Illiterate & \multicolumn{6}{|c|}{ Reference } \\
\hline & Less than high school diploma & 0.60 & 0.023 & $(0.39-0.93)$ & 1.45 & 0.328 & $(0.68-3.07)$ \\
\hline & High school diploma or higher & 0.44 & 0.045 & $(0.19-1.01)$ & 9.33 & 0.013 & $(1.61-54.08)$ \\
\hline
\end{tabular}

\section{Discussion}

Overall five-year survival rate of patients with Gastric cancer was estimated at $16 \%$ which is along with the survival rate estimated in Tehran at $16.33 \%$ (9) and other provinces of Iran at $15 \%$ (11). However, it was lower than the results reported in the studies carried out in other countries like the US (13), Japan (14), and Taiwan (15). This low survival rate could be because most patients with Gastric cancer are diagnosed at a stage when conventional therapies such as gastrectomy, chemotherapy, or radiation therapy are not effective to increase the patients' survival 
(9). Another explanation could be that we conducted this retrospective study in a situation that the patients were diagnosed many years before, when the diagnosis and the treatment protocol were not as advanced as the current time. These findings are consistent with previous reports suggesting that poor diagnosis is the greatest challenge to Gastric cancer patients' survival in Iran (11).

In our previous study, by designing a predictive model of survival time for patients suffering from gastric cancer by data mining techniques using genetic algorithm and logistic regression, it was shown that sex, age, age at diagnosis time, and family history of gastric cancer are associated with patients' survival (15). However, we aimed to determine GC patients' survival with available data and classic statistical methods. In multiple regression analysis of this study, sex is shown to be a strong predictor of survival, and women had statistically higher survival rates than men. The results are also consistent with studies reported better survival rates for women (16-18). However, some studies have reported a higher death hazard for women $(9,19-21)$. Older age at diagnosis in the present study could increase death hazard, and this decremental effect of age on survival proportion has been also found in other parts of Iran $(9,11,17)$ and some other countries $(22,23)$.

Positive family history of gastric cancer and having ethnicity other than Fars increased the death hazard ratio but these differences were not statistically significant. Some studies conducted in the other part of Iran have also reported no effect on survival rate by ethnicity and family history $(17,24)$. Ghadimi et al., however, reported family history as a significant predictor of survival time in northern Iran (18). The survival rate of those who had a history of smoking or addiction was higher than those who had not. Nevertheless, some studies, reported lower survival rates in smokers $(9,24)$, none of which were statistically significant.

Owing to different numbers of missing data in each independent variable, multiple regression was limited to 77 observations. However, the results of univariate and multiple regression were not considerably different except for the level of education. According to the univariate analysis, patients with a higher level of education would have a higher survival rate. Nonetheless, based on multiple regression, this association was reversed. One explanation for this could be that in univariate analysis, the association between the level of education and survival rate is, at least in some part, confounded. For example, the mean of age is lower in patients with under high school diploma education levels in comparison to illiterates. However, we have no clear explanation why after adjusting other variables, the survival of patients with higher education is less than those with lower education. Maybe, the fitted regression model suffers from a lack of adjustment for some unmeasured variables like location or stage of tumor or residential area.

\section{Limitation of study}

A lack of a cancer registry in Khorasan Razavi province makes a huge obstacle to determine any features of Gastric Cancer in this province, and unfortunately, the results of this retrospective study were affected by lacking of adequate data about the disease stage.

\section{Conclusions}

The current study showed that older age at diagnosis time, male sex, coronary artery disease, diabetes mellitus, and hypertension and also the combination of these comorbidities are factors which can negatively influence the survival rate of gastric cancer patients. These can be applied to improve gastric cancer screening and treatment guidelines. Cancer registry in high incidence areas such as Mashhad might help conduct this study on a larger scale and achieve a more accurate estimation of the survival status and related factors.

\section{Acknowledgments:}

The authors gratefully acknowledge the financial support for this work provided by Mashhad University of Medical Sciences (Grant no: 941320).

\section{Conflict of Interest:}

There is no conflict of interest to be declared.

\section{Authors' contributions:}

Conception or design of the work (ZY, LG), Acquisition of data (ZY, NF, AG, ZD, RH), Analysis or interpretation of data (ZY, HHM), Drafting the manuscript (All authors except LG), Revising the manuscript (ZY, LG), Accountable for all aspects of the work (All authors). All authors read and approved the final manuscript. 


\section{References:}

1) Erratum: Global cancer statistics 2018: GLOBOCAN estimates of incidence and mortality worldwide for 36 cancers in 185 countries. CA: A Cancer Journal for Clinicians. 2020;70(4):313. doi: $10.3322 /$ caac. 21609 .

2) Sadjadi A, Malekzadeh R, Derakhshan MH, Sepehr A, Nouraie M, Sotoudeh M, et al. Cancer occurrence in Ardabil: results of a population-based cancer registry from Iran. International journal of cancer. 2003;107(1):113-8. Epub 2003/08/20. doi: 10.1002/ijc.11359. PMID: 12925965.

3) Babaei M, Mousavi S, Malek M, Tosi G, Masoumeh Z, Danaei N, et al. Cancer occurrence in Semnan Province, Iran: results of a population-based cancer registry. Asian Pacific journal of cancer prevention: APJCP. 2005;6(2):159-64. Epub 2005/08/17. PMID: 16101326.

4) Yazdizadeh B, Jarrahi AM, Mortazavi H, Mohagheghi MA, Tahmasebi S, Nahvijo A. Time trends in the occurrence of major GI cancers in Iran. Asian Pacific journal of cancer prevention: APJCP. 2005;6(2):1304. Epub 2005/08/17. PMID: 16101320.

5) Amirkhah R, Naderi-Meshkin H, Mirahmadi M, Allahyari A, Sharifi HR. Cancer statistics in Iran: Towards finding priority for prevention and treatment. 2017. 2017;3(2):12. Epub 2017-10-25. doi: 10.15562/tcp.42.

6) Jemal A, Bray F, Center MM, Ferlay J, Ward E, Forman D. Global cancer statistics. CA: a cancer journal for clinicians. 2011;61(2):69-90. Epub 2011/02/08. doi: 10.3322/caac.20107. PMID: 21296855.

7) Sadjadi A, Nooraie M, Ghorbani A, Alimohammadian M, Zahedi MJ, Darvish-Moghadam S, et al. The incidence of prostate cancer in Iran: results of a population-based cancer registry. Archives of Iranian medicine. 2007;10(4):481-5. Epub 2007/10/02. doi: 07104/aim.0011. PMID: 17903053.

8) Derakhshan MH, Malekzadeh R, Watabe H, Yazdanbod A, Fyfe V, Kazemi A, et al. Combination of gastric atrophy, reflux symptoms and histological subtype indicates two distinct aetiologies of gastric cardia cancer. Gut. 2008;57(3):298-305. Epub 2007/10/30. doi: 10.1136/gut.2007.137364. PMID: 17965056.

9) Zare A, Mahmoodi M, Mohammad K, Zeraati H, Hosseini M, Holakouie Naieni K. Factors Affecting the Survival of Patients with Gastric Cancer Undergone Surgery at Iran Cancer Institute: Univariate and Multivariate Analyses. Iranian journal of public health. 2014;43(6):800-8. Epub 2015/06/26. PMID: 26110151; PMCID: PMC4475599.

10) Ding Y-B, Chen G-Y, Xia J-G, Zang X-W, Yang H-Y, Yang L, et al. Correlation of tumor-positive ratio and number of perigastric lymph nodes with prognosis of patients with surgically-removed gastric carcinoma. World J Gastroenterol. 2004;10(2):182-5. doi: 10.3748/wjg.v10.i2.182. PMID: 14716818, PMCid: PMC4716999

11) Veisani Y, Delpisheh A. Survival rate of gastric cancer in Iran; a systematic review and meta-analysis. Gastroenterology and hepatology from bed to bench. 2016;9(2):78-86. Epub 2016/04/22. PMID: 27099666; PMCID: PMC4833845.

12) Goshayeshi L, Hoseini B, Yousefli Z, Khooie A, Etminani K, Esmaeilzadeh A, et al. Predictive model for survival in patients with gastric cancer. Electronic physician. 2017;9(12):6035-42. Epub 2018/03/22. doi: 10.19082/6035. PMID: 29560157; PMCID: PMC5843431.

13) Schwarz RE, Zagala-Nevarez K. Recurrence patterns after radical gastrectomy for gastric cancer: prognostic factors and implications for postoperative adjuvant therapy. Annals of surgical oncology. 2002;9(4):394-400. Epub 2002/05/03. PMID: 11986192.

14) Adachi Y, Tsuchihashi J, Shiraishi N, Yasuda K, Etoh T, Kitano S. AFP-producing gastric carcinoma: multivariate analysis of prognostic factors in 270 patients. Oncology. 2003;65(2):95-101. Epub 2003/08/22. doi: 10.1159/000072332. PMID: 12931013.

15) Wang CS, Hsieh CC, Chao TC, Jan YY, Jeng LB, Hwang TL, et al. Resectable gastric cancer: operative mortality and survival analysis. Chang Gung medical journal. 2002;25(4):216-27. Epub 2002/06/25. PMID: 12079155.

16) Canyilmaz E, Soydemir G, Serdar L, Uslu GH, Sahbaz A, Colak F, et al. Evaluation of prognostic factors and survival results in gastric carcinoma: single center experience from Northeast Turkey. International journal of clinical and experimental medicine. 2014;7(9):2656-66. Epub 2014/10/31. PMID: 25356123; PMCID: PMC4211773.

17) Yazdani-Charati J, Janbabaei G, Etemadinejad S, Sadeghi S, Haghighi F. Survival of patients with stomach adenocarcinoma in North of Iran. Gastroenterology and hepatology from bed to bench. 2014;7(4):211-7. Epub 2014/10/08. PMID: 25289135; PMCID: PMC4185875.

18) Ghadimi M, Mahmoodi M, Mohammad K, Zeraati H, Rasouli M, Sheikhfathollahi M. Family history of the cancer on the survival of the patients with gastrointestinal cancer in northern Iran, using frailty models. 
BMC gastroenterology. 2011;11:104. Epub 2011/10/04. doi: 10.1186/1471-230x-11-104. PMID: 21961837; PMCID: PMC3217861.

19) Otsuji E, Yamaguchi T, Sawai K, Sakakura C, Okamoto K, Takahashi T. Regional lymph node metastasis as a predictor of peritoneal carcinomatosis in patients with Borrmann type IV gastric carcinoma. The American journal of gastroenterology. 1999;94(2):434-7. Epub 1999/02/18. doi: 10.1111/j.15720241.1999.873_b.x. PMID: 10022642.

20) Yagi Y, Seshimo A, Kameoka S. Prognostic factors in stage IV gastric cancer: univariate and multivariate analyses. Gastric cancer : official journal of the International Gastric Cancer Association and the Japanese Gastric Cancer Association. 2000;3(2):71-80. Epub 2002/05/02. PMID: 11984714.

21) Koizumi W, Kurihara M, Tanabe S, Kondo I, Yamazaki I, Nonaka M, et al. Advantages of Japanese response criteria for estimating the survival of patients with primary gastric cancer. Gastric cancer : official journal of the International Gastric Cancer Association and the Japanese Gastric Cancer Association. 1999;2(1):14-9. Epub 2002/04/17. doi: 10.1007/s101209900021. PMID: 11957065.

22) Enzinger PC, Mayer RJ. Gastrointestinal cancer in older patients. Semin Oncol. 2004;31(2):206-19. Epub 2004/04/28. PMID: 15112151.

23) Saidi RF, Bell JL, Dudrick PS. Surgical resection for gastric cancer in elderly patients: is there a difference in outcome? The Journal of surgical research. 2004;118(1):15-20. Epub 2004/04/20. doi: 10.1016/s00224804(03)00353-6. PMID: 15093711.

24) Baeradeh NA, Lotfi MH, Fallahzadeh H, Kargar S, Salman Roghani H. Survival Rate of Patients with Stomach Cancer and its Effective Factors in Yazd Province. Journal of Community Health Research. 2015;3(4):278-87. 